\title{
Desenvolvimento Neuropsicomotor de lactentes prematuros vinculados à Clínica da Mulher de Chapecó
}

\author{
Neuropsychomotor Development of preterm infants linked to the Woman's Clinic in Chapecó City
}

Cristina Giaretta ${ }^{1}$, Simone Marlise Becker ${ }^{2}$, Rubia do Nascimento Fuentefria ${ }^{3}$

\begin{abstract}
RESUMO
Objetivo. Avaliar o Desenvolvimento Neuropsicomotor (DNPM) de lactentes prematuros e com baixo peso. Método. Estudo transversal, com população de 32 lactentes vinculados à Clínica da Mulher, Chapecó, SC. Destes, foram avaliados 22 com idade cronológica de 6 a 10 meses e 29 dias, por meio da Escala de Desenvolvimento Psicomotor da Primeira Infância Brunet-Lèzine. Para conhecer a história do período pré, peri e pós-natal aplicou-se um questionário aos pais. Resultados. Em relação aos 22 lactentes avaliados, a área de desenvolvimento que mais se destacou, em termos de alerta para atraso ou atraso já instalado, foi a coordenação óculo-motriz. Cinco lactentes da faixa etária de 6 a 7 meses e 29 dias e 5 lactentes da faixa etária de 8 a 10 meses e 29 dias apresentaram classificação normal baixo ou inferior nesta área. A maioria $(\mathrm{n}=16)$ dos lactentes de ambas as faixas etárias necessitaram de medidas de urgência para respirar e desenvolveram complicaçóes respiratórias logo após o parto $(n=11)$. Estas condiçóes biológicas de risco foram somadas à prematuridade e ao baixo peso. Conclusáo. Os resultados encontrados reforçam a necessidade de identificar precocemente os sinais de atrasos no desenvolvimento de lactentes vulneráveis, possibilitando a realização de um programa de intervenção precoce.
\end{abstract}

Unitermos. Lactente Prematuro, Baixo peso ao nascer, Avaliação, Desempenho Psicomotor.

Citaçáo. Giaretta C, Becker SM, Fuentefria RN. Desenvolvimento Neuropsicomotor de lactentes prematuros vinculados à Clínica da Mulher de Chapecó.

\begin{abstract}
Objective. To evaluate the Neuropsychomotor Development (NPMD) in preterm infants and low birth weight. Method. Cross-sectional study, with population of 32 infants linked to the Woman's Clinic in Chapecó City. Of these, 22 were evaluated with chronological age of 6-10 months and 29 days, through Brunet-Lezine Scale. To know the history of prenatal, perinatal and postnatal period was applied a questionnaire to the children's parents. Results. In relation to the 22 evaluated infants, the development area that more stood out, in alert terms for delay or delay already installed, it was the oculomotor coordination. Five infants of the age group from 6 to 7 months and 29 days and 5 infants in the age group from 8 to 10 months and 29 days presented normal low classification or lower than normal in this area. Most of the infants $(n=16)$ of both age groups needed of urgent measures to breathe and they had respiratory complications after birth $(\mathrm{n}=11)$. These biological risk conditions were added to the prematurity and the underweight. Conclusion. The found results reinforce the need to identify earlier the signs of delay in the development of vulnerable infants, making possible the accomplishment of a program of precocious intervention.
\end{abstract}

Keywords. Premature, Low Birth Weight, Evaluation, Psychomotor Performance.

Citation. Giaretta C, Becker SM, Fuentefria RN. Neuropsychomotor Development of preterm infants linked to the Woman's Clinic in Chapecó City.
Trabalho realizado na Universidade Comunitária da Regiáo de Chapecó, UNOCHAPECÓ, Chapecó-SC, Brasil.

1. Fisioterapeuta Graduada pela Universidade Comunitária da Regiáo de Chapecó - UNOCHAPECÓ, Chapecó-SC, Brasil.

2. Fisioterapeuta Graduada pela Universidade Comunitária da Regiâo de Chapecó - UNOCHAPECÓ, Chapecó-SC, Brasil.

3. Fisioterapeuta, Mestranda do Programa de Pós-Graduação em Saúde da Criança e do Adolescente da Universidade Federal do Rio Grande do Sul UFRGS, Porto Alegre-RS, Brasil.
Endereço para correspondência: Rubia N Fuentefria Serviço de Neonatologia do Hospital de

Clínicas de Porto Alegre, sala 1123

Ramiro Barcelos, 2350, Santa Cecília CEP 90035-903, Porto Alegre-RS, Brasil.

E-mail: rubianascif@gmail.com 


\section{INTRODUÇÃO}

O Desenvolvimento Neuropsicomotor (DNPM) caracteriza-se pela capacidade do indivíduo em adquirir funçôes cada vez mais complexas no decorrer do tempo. Estas, por sua vez, resultam da interação entre as características biológicas e ambientais, as quais irão permitir um aprimoramento na conduta sensorial, motora, emocional, cognitiva, de linguagem e de aprendizagem ${ }^{1}$.

Porém, existem alguns fatores que podem colocar em risco o DNPM típico de lactentes. Estes fatores constituem uma série de condições biológicas e/ou ambientais que poderão aumentar a probabilidade da existência de déficits no desenvolvimento. As condiçôes biológicas compreendem os problemas maternos como a hipertensão e o diabetes, bem como a prematuridade, o baixo peso ao nascer, principalmente o peso inferior a $1.500 \mathrm{~g}$, a hipóxia neonatal grave e a hiperbilirrubinemia ${ }^{2}$. Dentre estas condiçóes, a prematuridade (idade gestacional inferior a 37 semanas) e o baixo peso ao nascer (inferior a $2.500 \mathrm{~g}$ ) são considerados importantes fatores de risco para atraso no $\mathrm{DNPM}^{3,4}$.

O lactente prematuro possui um desenvolvimento em um ritmo mais lento, já que não atinge o grau completo do tônus muscular flexor visto em recém-nascidos a termo, ocorrendo um desequilíbrio entre os grupos musculares flexores e extensores. Esse desequilíbrio pode interferir no controle da cabeça, na simetria, no equilíbrio da postura sentada, na aquisição de habilidades e na coordenação bilateral ${ }^{5}$. Os lactentes prematuros possuem um maior risco de desenvolver doenças respiratórias, doenças da membrana hialina, hiperbilirrubinemia, hipocalcemia, anemia e outras alteraçóes que afetam a saúde e consequentemente seu desenvolvimento ${ }^{6}$. Além disso, o nascimento com baixo peso e principalmente com muito baixo peso (inferior a $1.500 \mathrm{~g}$ ) podem comprometer algumas áreas do desenvolvimento, desencadeando problemas de linguagem, de aprendizagem e de coordenação visomotora ${ }^{7}$.

Os avanços científicos e tecnológicos das últimas décadas associaram-se a grandes mudanças na assistência obstétrica e neonatal, promovendo a redução da mortalidade neonatal e um aumento relevante nas taxas de sobrevida de lactentes prematuros e de baixo peso. Todavia, o aumento nas taxas de sobrevida não garante que estes irão ter um desenvolvimento normal ${ }^{8}$.
Para tentar minimizar possíveis problemas no desenvolvimento destes lactentes, torna-se necessária a identificação precoce de desvios e a intervenção em tempo oportuno ${ }^{2}$. Neste sentido, o fisioterapeuta tem um papel fundamental no diagnóstico e tratamento de problemas no desenvolvimento, estimulando o potencial de desenvolvimento global, fazendo com que a criança com atraso se torne apta a responder às suas necessidades e às do seu meio?.

Considerando o exposto e a importância da avaliação e intervenção precoce mediante a presença de fatores de risco biológicos, este estudo teve como objetivos avaliar o desenvolvimento de lactentes prematuros e com baixo peso na faixa etária dos 6 aos 10 meses de idade cronológica, vinculados à Clínica da Mulher do município de Chapecó-SC, na área postural, de coordenação óculo-motriz, da linguagem e social e analisar os fatores biológicos que possam estar influenciando esse desenvolvimento.

\section{MÉTODO}

\section{Amostra}

Este estudo caracterizou-se como uma pesquisa transversal, com abordagem quantitativa dos resultados. A população do estudo foi constituída de lactentes prematuros e com baixo peso nascidos no período de abril a setembro de 2009, totalizando 32 lactentes. Considerando os critérios de inclusão e exclusão descritos abaixo, a amostra foi composta de 22 lactentes com idade cronológica de 6 meses a 10 meses e 29 dias, vinculados à Clínica da Mulher localizada no município de Chapecó - SC. Os lactentes avaliados não recebiam atendimentos de Estimulação Precoce.

Os critérios de inclusão elegíveis para o estudo foram: idade gestacional inferior a 37 semanas (prematuros), peso ao nascer inferior à $2500 \mathrm{~g}$ (baixo peso e muito baixo peso), idade cronológica de 6 meses a 10 meses e 29 dias, residentes no município de Chapecó, SC e usuários do sistema único de saúde (SUS). Os critérios de exclusão foram: número de consultas pré-natais inferior a três, lactentes com diagnóstico de mal-formaçôes congênitas, patologias inatas do metabolismo, tentativa de abortos, ausência e mudança de endereço para contato, não aceitação para participação no estudo e o não comparecimento no dia e horário agendado para a avaliação. 
Esta pesquisa foi aprovada pelo Comitê de Ética da Universidade Comunitária da Região de Chapecó Unochapecó, com parecer no 264/09. Seguindo as normas éticas da Resolução nº 196/96 do Conselho Nacional de Saúde, o responsável por cada lactente assinou um Termo de Consentimento Livre e Esclarecido, autorizando o estudo.

\section{Procedimento}

Como instrumento para coleta de dados foi utilizado um questionário adaptado ${ }^{10}$ que abordou informaçóes relacionadas ao período gestacional e ao parto. Este questionário foi composto por perguntas fechadas que foram respondidas pela mãe. Também foi utilizada a Escala de Desenvolvimento Psicomotor da Primeira Infância Brunet-Lèzine ${ }^{11}$, adaptada de acordo com as condiçóes culturais do Brasil ${ }^{10}$.

A Escala Brunet-Lèzine avalia lactentes com faixa etária entre 0 e 30 meses de idade e envolve uma avaliação complexa do controle postural e motricidade, linguagem, coordenação óculo-motriz, relaçôes sociais e pessoais. Apresenta 10 itens a serem avaliados a cada mês, sendo que os seis primeiros itens referem-se a testes de desenvolvimento postural, de coordenação óculo-motriz, de linguagem e social; já os quatro últimos itens são questôes direcionadas à mãe do lactente ${ }^{11}$.

O kit de avaliação da Escala Brunet-Lèzine incluiu objetos que foram apresentados às crianças pelo examinador com a finalidade de observar suas reaçóes e assim responder às questôes dos 6 primeiros itens de testagem de cada mês. Os objetos foram os seguintes: 10 cubos de madeira, vermelho vivo, de $2,5 \mathrm{~cm}$ de aresta; uma xícara de alumínio, de $7,5 \mathrm{~cm}$ de altura e $8,5 \mathrm{~cm}$ de diâmetro; uma colher de alumínio, de $16 \mathrm{~cm}$ de comprimento; uma campainha metálica brilhante (sino) com cabo de madeira de $6 \mathrm{~cm}$ de comprimento e sino metálico de $5,5 \mathrm{~cm}$ de altura e gargalo de $6 \mathrm{~cm}$ de diâmetro; um chocalho pequeno, colorido e de material plástico com $18 \mathrm{~cm}$ de comprimento; um anel de madeira, vermelho, de $13 \mathrm{~cm}$ de diâmetro, amarrado a uma fita preta de $25 \mathrm{~cm}$ de comprimento e $1 \mathrm{~cm}$ de largura; um espelho de $30 \mathrm{~cm}$ x 25 $\mathrm{cm}$; um pano quadrado com $39 \mathrm{~cm}$ de face (tecido não transparente); um botão de polímero, preto, de $8 \mathrm{~mm}$ de diâmetro; um lápis de cor azul, de $10 \mathrm{~cm}$ de compri- mento; um bloco de papel, de $20 \mathrm{~cm}$ x $13 \mathrm{~cm}$; um frasco de plástico, de $9 \mathrm{~cm}$ de altura com gargalo de $2 \mathrm{~cm}$ de diâmetro ${ }^{11}$.

O período da coleta de dados foi de fevereiro a março de 2010. A coleta foi realizada a partir de um mapeamento dos prontuários dos lactentes acompanhados na Clínica da Mulher, localizada no município de Chapecó - SC, para verificar o número de lactentes nascidos prematuros e com baixo peso e entáo definir a amostra. A partir deste mapeamento entrou-se em contato com os representantes legais (mães) dos lactentes.

O questionário adaptado ${ }^{10}$ foi aplicado pelas pesquisadoras ao responsável pelo lactente. Após a aplicação do questionário foi realizada a avaliação do lactente por meio da Escala Brunet-Lèzini nesta mesma data. Cada avaliação durou aproximadamente 30 minutos.

A escolha do local para aplicação do questionário e da avaliação foi definida mediante as condições de deslocamento do responsável pelo lactente. Desta forma, os instrumentos foram aplicados tanto na Clínica da $\mathrm{Mu}$ lher, na Clínica Escola de Fisioterapia da Unochapecó, bem como no domicílio dos próprios lactentes. Mesmo sendo em diferentes locais, a aplicação da escala seguiu as normas descritas no Manual ${ }^{11}$.

Posteriormente à avaliação, a pesquisadora preencheu os itens da escala correspondentes aos meses avaliados, encontrou a pontuação para cada área e na sequência converteu os números em idades de desenvolvimento, para então calcular os quocientes do desenvolvimento, considerando que a idade cronológica dos lactentes foi corrigida antes da realização dos cálculos. A idade corrigida representa o ajuste da idade cronológica em relação ao grau de prematuridade. A utilização da idade corrigida na avaliação do desenvolvimento de pré-termos evita a subestimação destes lactentes na comparação com a população de referência, permitindo uma avaliação mais precisa. Os valores numéricos encontrados nos quocientes de desenvolvimento foram relacionados a níveis motores, permitindo classificar as habilidades avaliadas (Postural, Coordenação óculo-motriz, Linguagem, Sociabilidade e Global) em padrões: Muito Superior (130 ou mais), Superior (120-129), Normal Alto (110-119), Normal Médio (90-109), Normal Baixo (80-89), Inferior (70-79) e Muito Inferior (69 ou menos) $)^{10,11}$. 


\section{Análise dos Dados}

Para fins de uma análise mais homogênea dos dados quanto ao desenvolvimento dos lactentes, os mesmos foram agrupados em faixas etárias, sendo de 6 a 7 meses e 29 dias e de 8 a 10 meses e 29 dias. Os dados foram analisados de forma descritiva e apresentados através de tabelas contendo frequências, médias, desvio padrão, valores mínimos e máximos e percentuais.

\section{RESULTADOS}

Quanto às características da amostra no que se refere à frequência e ao sexo foram avaliados 22 lactentes, sendo 10 do sexo masculino e 12 do sexo feminino. Quanto à frequência simples das idades cronológicas participaram 8 lactentes entre 6 a 7 meses e 29 dias e 14 lactentes entre 8 a 10 meses e 29 dias.

Dos fatores associados ao DNPM, 8 lactentes apresentaram idade gestacional ao nascimento variando de 35 a 36 semanas; 13 de 31 a 34 semanas e um igual a
30 semanas. A média da variável idade gestacional foi de 34 semanas. Quanto ao peso ao nascer, apenas um caso da amostra apresentou peso inferior a $1.000 \mathrm{~g}$, os demais nasceram com peso entre 1.500 e $2.500 \mathrm{~g}$. A média do peso ao nascer foi de $2.011 \mathrm{~g}$, o valor mínimo foi de $945 \mathrm{~g}$ e o valor máximo de $2.370 \mathrm{~g}$. Em relação ao Apgar no $1^{\circ}$ minuto, o valor médio foi de 7,45, com valor mínimo de 2 e máximo de 9; 2 casos apresentaram Apgar inferior a 7. Já no $5^{\circ}$ minuto, o valor médio do Apgar foi de 8,72 , com valor mínimo de 7 e máximo de 10 .

A Tabela 1 apresenta as condições ligadas ao período pré e peri-natal do lactente. Quanto às ocorrências gestacionais, observa-se que em 13 casos do presente estudo foi relatada intercorrência pelo responsável. Dentre as citadas, a mais frequente foi a pressão alta $(n=5)$. Em relação ao tipo de parto, a maioria $(n=14)$ relatou parto cesariano. Quanto aos casos de gemelaridade, destaca-se uma frequência elevada, já que quase a metade da amostra ( $\mathrm{n}=10 ; 5$ casais) foi constituída de lactentes gêmeos.

Tabela 1

Condiçóes ligadas ao nascimento da criança

\begin{tabular}{|c|c|c|c|}
\hline Período Pré e Perinatal & Classificação & Frequência & Percentual \\
\hline \multirow[t]{8}{*}{ Ocorrências Gestacionais } & Pressão alta & 5 & 22,7 \\
\hline & Infecção & 1 & 4,5 \\
\hline & Ameaça de aborto & 1 & 4,5 \\
\hline & Sangramento exagerado & 2 & 9,1 \\
\hline & Infecção Urinária & 1 & 4,5 \\
\hline & Dilatação pélvica precoce & 2 & 9,1 \\
\hline & Diabetes Gestacional & 1 & 4,5 \\
\hline & Nenhuma intercorrência & 10 & 45,4 \\
\hline \multirow[t]{3}{*}{ Tipo de parto } & Cesárea & 14 & 63,63 \\
\hline & Normal & 8 & 36,36 \\
\hline & Normal / Fórceps & 0 & 0 \\
\hline Gemelaridade & Sim & 10 & 45,45 \\
\hline $\begin{array}{c}\text { Urgência no } \\
\text { momento do parto } \\
\text { para o bebê respirar }\end{array}$ & $\operatorname{Sim}$ & 16 & 72,72 \\
\hline \multirow{6}{*}{$\begin{array}{l}\text { Complicaçóes logo } \\
\text { após nascimento }\end{array}$} & Problemas respiratórios & 11 & 50 \\
\hline & Problemas cardíacos & 2 & 9 \\
\hline & Infecçôes & 4 & 18 \\
\hline & Convulsōes & 1 & 4,5 \\
\hline & Hipoglicemia & 2 & 9 \\
\hline & Refluxo gastro-esofágico & 2 & 9 \\
\hline
\end{tabular}


Em se tratando dos casos de urgência no momento do parto e complicaçóes, percebem-se na Tabela 1 números elevados de lactentes que precisaram de alguma forma de ajuda externa para respirar $(n=16)$, bem como de complicaçôes após o nascimento $(\mathrm{n}=21)$, sendo a complicação respiratória a mais frequente $(\mathrm{n}=11)$.

Em relação à avaliação do DNPM, a Tabela 2 expressa os valores das variáveis obtidas pela aplicação da Escala Brunet-Lèzini dos 8 lactentes do estudo que se enquadraram na faixa etária de 6 a 7 meses e 29 dias. Os resultados descritos demonstram que a idade cronológica (IC) média da amostra na avaliação foi de 6,2 meses e a idade cronológica corrigida (ICC) média foi de 4,8 meses. As médias das idades de desenvolvimento (ID) nas áreas postural, de coordenação óculo-motriz, da linguagem, da sociabilidade e global variaram de 4,9 a 6,2 meses, sendo valores superiores à média da ICC. Porém, os valores mínimos encontrados nas ID oscilaram de 3,6 a 4,3 meses, sendo estes inferiores à média da ICC. Os valores máximos variaram de 7 a 8,3 meses. As médias dos quocientes de desenvolvimento (QD) do presente estudo apresentaram valores de 102,1 a 131,4. Os valores mínimos encontrados para os quocientes de desenvolvimento foram de 72 a 81,2 e os valores máximos variaram de 151 a 169,3 .

A Tabela 3 apresenta os valores das variáveis obtidas pela aplicação da Escala Brunet-Lèzini dos 14 lactentes do estudo que se enquadraram na faixa etária de 8 a 10 meses e 29 dias. Os resultados descritos demonstram que a IC média da amostra na avaliação foi de 9,5 meses e a ICC média foi de 8,1 meses. As médias das ID nas áreas postural, de coordenação óculo-motriz, da linguagem, da sociabilidade e global variaram de 7,9 a 8,8 meses, sendo que apenas na área da coordenação óculo-motriz o valor ficou inferior à ICC. Porém, os valores mínimos encontrados nas idades de desenvolvimento oscilaram de 5,6 a 7 meses, sendo estes inferiores à média da ICC. Já os valores máximos variaram de 10,4 a 15 meses. As médias dos QD apresentaram valores de 98,6 a 109,2. Os valores mínimos encontrados para os quocientes de desenvolvimento foram de 72,1 a 76,6 e os valores máximos variaram de 119 a 156,9.

As frequências dos lactentes com os níveis motores (classificação) obtidos a partir do cálculo dos QD, em cada área do DNPM avaliada, estão apresentadas nas Tabelas 4 e 5 . A classificação normal baixo é considera-

Tabela 2

Valores das variáveis obtidas pela aplicação da Escala Brunet-Lèzine $e^{13}$ na faixa etária de 6 a 7 meses e 29 dias

\begin{tabular}{|c|c|c|c|c|}
\hline & Média & Desvio Padráo & Mínimo & Máximo \\
\hline $\begin{array}{c}\text { Idade Cronológica } \\
\text { em meses }\end{array}$ & 6,2 & 0,25 & 6,0 & 6,6 \\
\hline $\begin{array}{c}\text { Idade Cronológica } \\
\text { Corrigida } \\
\text { em meses }\end{array}$ & 4,8 & 0,30 & 4,5 & 5,3 \\
\hline \multicolumn{5}{|l|}{$\begin{array}{c}\text { Idades de } \\
\text { Desenvolvimento }\end{array}$} \\
\hline Postural & 6,0 & 1,48 & 4,3 & 8,3 \\
\hline $\begin{array}{l}\text { Coordenação } \\
\text { óculo-motriz }\end{array}$ & 4,9 & 1,65 & 3,6 & 7,5 \\
\hline Linguagem & 6,2 & 1,16 & 4,0 & 7,0 \\
\hline Sociabilidade & 5,9 & 1,40 & 4,3 & 8,0 \\
\hline Global & 5,5 & 1,41 & 3,7 & 7,8 \\
\hline \multicolumn{5}{|l|}{$\begin{array}{c}\text { Quocientes de } \\
\text { Desenvolvimento }\end{array}$} \\
\hline Postural & 125,4 & 30,5 & 81,2 & 169,3 \\
\hline $\begin{array}{l}\text { Coordenação } \\
\text { óculo-motriz }\end{array}$ & 102,1 & 30,2 & 72 & 151,0 \\
\hline Linguagem & 131,4 & 30,4 & 75,0 & 155,5 \\
\hline Sociabilidade & 123,3 & 28,6 & 81,0 & 165,4 \\
\hline Global & 115,9 & 28,4 & 74,0 & 159,1 \\
\hline
\end{tabular}


Valores das variáveis obtidas pela aplicação da Escala Brunet-Lèzine ${ }^{13}$ na faixa etária de 8 a 10 meses e 29 dias

\begin{tabular}{|c|c|c|c|c|}
\hline & Média & Desvio Padráo & Mínimo & Máximo \\
\hline $\begin{array}{c}\text { Idade Cronológica } \\
\text { em meses }\end{array}$ & 9,5 & 1,01 & 8,0 & 10,9 \\
\hline $\begin{array}{c}\text { Idade Cronológica } \\
\text { Corrigida } \\
\text { em meses }\end{array}$ & 8,1 & 1,15 & 6,5 & 9,7 \\
\hline \multicolumn{5}{|l|}{$\begin{array}{c}\text { Idades de } \\
\text { Desenvolvimento }\end{array}$} \\
\hline Postural & 8,8 & 2,31 & 5,6 & 15,0 \\
\hline $\begin{array}{l}\text { Coordenação } \\
\text { óculo-motriz }\end{array}$ & 7,9 & 1,25 & 5,7 & 10,4 \\
\hline Linguagem & 8,3 & 1,78 & 7,0 & 12,0 \\
\hline Sociabilidade & 8,5 & 1,73 & 6,0 & 13,5 \\
\hline Global & 8,3 & 1,44 & 5,9 & 11,9 \\
\hline \multicolumn{5}{|l|}{$\begin{array}{c}\text { Quocientes de } \\
\text { Desenvolvimento }\end{array}$} \\
\hline Postural & 109,2 & 25,0 & 72,1 & 156,9 \\
\hline $\begin{array}{l}\text { Coordenação } \\
\text { óculo-motriz }\end{array}$ & 98,6 & 16,6 & 72,5 & 119,0 \\
\hline Linguagem & 103,1 & 18,3 & 72,1 & 136,0 \\
\hline Sociabilidade & 106,9 & 23,7 & 72,1 & 143,0 \\
\hline Global & 103,4 & 17,4 & 76,6 & 127,0 \\
\hline
\end{tabular}

da sinal de alerta para atraso no desenvolvimento, já a classificação inferior e muito inferior demonstram uma condição de atraso instalado. As demais classificações são representativas de parâmetros de normalidade.

Em relação à faixa etária de 6 a 7 meses e 29 dias, apresentada na Tabela 4, encontrou-se um caso de alerta para atraso (classificação normal baixo) nas áreas postural, sociabilidade e global (o mesmo lactente apresentou alerta para atraso nestas três áreas), sendo que na linguagem este mesmo lactente apresentou classificação inferior, ou seja, uma condição de atraso nesta área. Outros 3 casos diferentes de alerta para atraso foram encontrados na área da coordenaçáo óculo-motriz. Em outro caso constatou- se atraso (classificação inferior) na área da coordenação óculo-motriz e no desenvolvimento global.

Tratando-se da faixa etária de 8 a 10 meses e 29 dias apresentada na Tabela 5, foi encontrado 1 caso com atraso (classificaçáo inferior) no desenvolvimento em todas as áreas e 3 diferentes casos com atraso em pelo menos duas áreas do desenvolvimento. As áreas que apresentaram mais lactentes com atraso no desenvolvimento foram a coordenação óculomotriz, postural e sociabilidade, cada uma com 3 casos de atraso. Já na classificação normal baixo, considerados lactentes com sinal de alerta para atraso no DNPM, foram encontrados 3 casos diferentes nas áreas da coordenação óculomotriz, linguagem, sociabilidade e desenvolvimento global.

Tabela 4

Frequência simples dos niveis motores de classificação encontrados a partir do Quociente de Desenvolvimento na faixa etária de 6 a 7 meses e 29 dias

\begin{tabular}{|c|c|c|c|c|c|c|c|}
\hline Áreas & Muito Superior & Superior & Normal Alto & Normal Médio & Normal Baixo & Inferior & Muito Inferior \\
\hline Postural & 4 & 1 & 1 & 1 & 1 & 0 & 0 \\
\hline $\begin{array}{l}\text { Coordenaçáo } \\
\text { óculo-motriz }\end{array}$ & 2 & 0 & 0 & 2 & 3 & 1 & 0 \\
\hline Linguagem & 5 & 0 & 1 & 1 & 0 & 1 & 0 \\
\hline Sociabilidade & 3 & 2 & 1 & 1 & 1 & 0 & 0 \\
\hline Global & 2 & 1 & 2 & 1 & 1 & 1 & 0 \\
\hline
\end{tabular}


Tabela 5

Frequência simples dos niveis motores de classificação encontrados a partir do Quociente de Desenvolvimento na faixa etária de 8 a 10 meses e 29 dias

\begin{tabular}{cccccccc}
\hline Áreas & Muito Superior & Superior & Normal Alto & Normal Médio & Normal Baixo & Inferior & Muito Inferior \\
\hline $\begin{array}{c}\text { Postural } \\
\begin{array}{c}\text { Coordenaçá́ } \\
\text { óculo-motriz }\end{array}\end{array}$ & 4 & 1 & 1 & 5 & 0 & 3 & 0 \\
Linguagem & 2 & 0 & 5 & 5 & 1 & 3 & 0 \\
Sociabilidade & 3 & 1 & 1 & 8 & 1 & 1 & 0 \\
Global & 0 & 1 & 2 & 4 & 2 & 2 & 0 \\
\hline
\end{tabular}

\section{DISCUSSÃO}

No presente trabalho, houve maior ocorrência de prematuridade moderada ( $\mathrm{n}=13)$, a qual pode ser definida quando o neonato é nascido entre 31 e 34 semanas de gestação ${ }^{12}$. Estudo realizado em um hospital público que teve como objetivo caracterizar as crianças prematuras nascidas na cidade de Patrocínio (MG), concluiu que, dos nascidos pré-termo com peso inferior a $2.500 \mathrm{~g}$, a ocorrência de prematuridade moderada fez-se presente em $60,8 \%$ de uma amostra de 59 crianças $^{13}$. A maior parte desta amostra foi constituída por lactentes com idade gestacional entre 31 a 34 semanas, dado este que também se fez presente em nosso estudo.

Procedimentos de urgência para o bebê respirar no momento do parto, nos prematuros moderados da nossa amostra, foram necessários em 7 casos. O desenvolvimento de problemas respiratórios após o parto acometeu a maioria $(n=11)$ dos lactentes, seguido das infecçóes. Lactentes moderadamente prematuros apresentam baixa taxa de mortalidade e podem desenvolver problemas como doença da membrana hialina e asfixia perinatal, problemas ligados à transfusão de sangue de reserva placentário e hiperbilirrubinemia ${ }^{14}$.

Ocorrências de lactentes considerados prematuros limítrofes também foram encontradas no estudo $(n=8)$. Este grupo de neonatos compreende os nascidos entre a $35^{\mathrm{a}}$ e a $36^{\mathrm{a}}$ semanas de gestação ${ }^{12}$. Destas ocorrências, 2 casos necessitaram de procedimento de urgência para respirar no momento do parto (oxigenioterapia) e outros 2 apresentaram como complicação a hipoglicemia. Um estudo de triagem realizado em um ambulatório especializado em São Paulo demonstrou que, na ocorrência de prematuridade limítrofe, as complicaçóes pós-natais mais comuns estão relacionadas aos problemas respiratórios principalmente nas primeiras 48 horas de vida, seguidas posteriormente da hiperbilirrubinemia ${ }^{15}$.

Em se tratando da classificação extremamente prematuro, o presente estudo encontrou somente 1 caso, o qual necessitou de procedimentos de urgência para respirar no momento do parto, desenvolvendo, posteriormente ao parto, problemas respiratórios. Os recém-nascidos prematuros extremos apresentam idade gestacional menor ou igual a 30 semanas e geralmente nascem com menos de $1.500 \mathrm{~g}$, apresentando, em decorrência dessa maior imaturidade, intercorrências mais frequentes e mais graves, como a retinopatia da prematuridade ${ }^{12}$. As asfixias perinatais, os problemas na manutenção da temperatura corpórea, a insuficiência respiratória, as crises de apnéia, as infecções adquiridas e a hemorragia intracraniana também são encontradas com mais frequência neste grupo de prematuros $^{14}$. Em uma pesquisa realizada na Unidade de Terapia Intensiva Pediátrica e Neonatal do Hospital Universitário da Universidade de São Paulo, a maioria dos bebês nascidos foi classificada como prematuros extremos, com idade gestacional inferior a 30 semanas e, segundo o peso ao nascer, apresentaram classificaçáo baixo peso extremo (igual ou inferior $1.000 \mathrm{~g}$ ), denotando um alto risco para apresentar morbidades e um longo período de internação ${ }^{16}$.

Em relação ao peso ao nascer, observou-se no estudo que somente um lactente apresentou peso de $945 \mathrm{~g}$, sendo este representativo de classificação baixo peso extremo (peso inferior a $1000 \mathrm{~g}$ ). Este lactente também se enquadrou na classificação extremamente prematuro. Todos os demais nasceram com peso entre $1.500 \mathrm{~g}$ e $2.500 \mathrm{~g}$, sendo considerados recém-nascidos de baixo peso (peso inferior a $2.500 \mathrm{~g}^{17}$ ). Em um estudo realizado com prematuros, onde se comparou o grupo de prematuros mo- 
derados e o grupo de prematuros extremos, observou-se que quanto maior o índice de prematuridade, menores os quocientes de desenvolvimento, em específico o da coordenação óculo-motriz ${ }^{18}$. Quanto mais baixo o peso de nascimento, maior a probabilidade das crianças desenvolverem problemas de desenvolvimento com sequelas de difícil reversão. Lactentes pré-termo e com peso abaixo de $1500 \mathrm{~g}$ tendem a apresentar significativamente problemas de deficiência mental, motora e deficiências sensoriais. E nos lactentes prematuros com peso inferior a $1000 \mathrm{~g}$, os problemas apresentados ao longo do curso do desenvolvimento podem estar relacionados ao crescimento físico e às áreas da cognição, aprendizagem e comportamental ${ }^{19}$.

Em termos de média, o Apgar da amostra demonstra valores superiores a 7 , tanto no primeiro como no quinto minuto, sendo que neste último houve um aumento em 1,27 quando comparado ao primeiro minuto, refletindo melhores condiçóes de vitalidade do grupo. Porém, ao observar o valor mínimo do primeiro minuto, percebe-se um número muito inferior a 7 (Apgar 2), demonstrando que um lactente ficou em situação de emergência médica. Entretanto, o valor mínimo do Apgar do quinto minuto sugere que houve uma recuperação do quadro do recémnascido, já que este valor passou de 2 (primeiro minuto) para 7 (quinto minuto). No presente estudo os dois lactentes que apresentaram Apgar 2 e 5 no $1^{\circ}$ minuto não apresentaram atraso no desenvolvimento neuropsicomotor. Estes lactentes apresentaram Apgar 7 e 8 no quinto minuto demonstrando que os mesmos conseguiram recuperar sua condição clínica. O índice de Apgar é considerado um indicador de risco para o neurodesenvolvimento quando for inferior a 6 no quinto minuto. Em um estudo envolvendo indicadores de risco para o DNPM, verificouse que, entre os fatores analisados, o índice de Apgar foi o único que mostrou correlação positiva com o exame neurológico alterado, uma vez que neonatos com Apgar de risco tiveram chance significativamente maior de apresentarem alguma manifestação neurológica ${ }^{20}$.

Destacam-se como fatores de risco associados à prematuridade a hipertensão gestacional, os sangramentos, a placenta prévia, o deslocamento prematuro da placenta, as infecçôes, dentre outros ${ }^{21}$. No presente estudo as ocorrências gestacionais mais encontradas foram a hipertensão, seguida de sangramentos exagerados, dilatação pélvica precoce, infecçóes, ameaça de aborto e diabetes. Em 10 casos do presente estudo náo foram relatadas ocorrências gestacionais. Muitas vezes o parto prematuro ocorre em mulheres sem fatores de risco, demonstrando que a relação direta entre os possíveis fatores de risco e o parto prematuro espontâneo é com frequência difícil de ser estabelecida ${ }^{21}$. Em concordância com nosso estudo, uma pesquisa que teve como objetivo caracterizar gestantes submetidas ao parto prematuro eletivo verificou que o sofrimento fetal anteparto, seguido pela hipertensão arterial foram as causas mais comumente encontradas como determinantes do parto prematuro ${ }^{22}$.

O tipo de parto encontrado com mais frequência no estudo foi o parto cesariano totalizando 14 partos, dado este que pode ser comparado ao resultado de um estudo realizado no município de Goiânia, que investigou os fatores de risco para mortalidade neonatal e pós-natal. Este estudo apontou que o parto cesariano, quando comparado ao parto espontâneo de lactentes prematuros e de baixo peso, mostrou ser um fator de proteção para a redução da mortalidade no período neonatal ${ }^{23}$.

A gemelaridade enquadra-se nos fatores de risco associados à prematuridade ${ }^{21}$. No presente estudo, 10 lactentes avaliados, de uma amostra de 22, eram gêmeos. A frequência de parto pré-termo é sabidamente maior nesta população. Gêmeos têm 5,4 vezes mais chance de nascer antes da $37^{\text {a }}$ semana de gestação ${ }^{24}$, estando suscetíveis às complicaçôes da prematuridade.

A necessidade de urgência no momento do parto para o bebê respirar foi necessária na maioria dos casos $(\mathrm{n}=16)$ e, dentre as complicaçóes que acometeram os lactentes do estudo logo após o nascimento, destacaram-se os problemas respiratórios. Lactentes prematuros possuem um maior risco de desenvolver doenças respiratórias e outras alteraçoos, que afetam a saúde e consequentemente seu desenvolvimento ${ }^{6}$. Outras complicações, como infecção, problemas cardíacos, convulsão e hipoglicemia, também foram encontradas. Em um estudo realizado com lactentes com idade gestacional inferior a 34 semanas também prevaleceram as complicaçóes respiratórias sendo elas as síndromes obstrutivas de vias aéreas, pneumonias e internamentos por problemas respiratórios ${ }^{25}$.

$\mathrm{Na}$ faixa etária de 6 a 7 meses e 29 dias (Tabela 2), as médias de ID nas áreas postural, de coordenação 
óculo-motriz, da sociabilidade e global foram superiores à média da ICC. Destas, a área da linguagem foi a que apresentou a maior média de idade de desenvolvimento, estando 1,5 meses (45 dias) à frente da ICC. Em termos de ID, nenhuma das áreas apresentou resultados inferiores à média da ICC, demonstrando que as crianças apresentam aquisiçóes psicomotoras apropriadas a esta média, dado que se comprova observando que as mesmas têm uma média de idade de desenvolvimento global de 0,8 meses (24 dias) acima da média da ICC. No entanto, em relação aos valores mínimos das ID nas áreas avaliadas, observa-se que todos ficaram abaixo da ICC, destacandose a área da coordenação óculo-motriz como sendo a que apresentou o menor valor $(3,6)$ e a maior diferença da ICC (1,2 meses ou 36 dias).

Analisando os QD do presente estudo (Tabela 2), referente à faixa etária de 6 a 7 meses e 29 dias, observase que todas as áreas psicomotoras estiveram dentro da normalidade. O nível motor da área da coordenação óculo-motriz e da área global foi classificado como normal médio e normal alto, respectivamente. Já na área postural e sociabilidade, a classificação obtida foi representativa do nível "superior", e na linguagem "muito superior". Entretanto, os valores mínimos dos QD, revelam classificação "normal baixo" na área da sociabilidade e postural e "inferior" nas demais áreas, demonstrando que mesmo com uma média dentro dos parâmetros da normalidade existiam casos com alerta para atraso e casos com atraso no desenvolvimento da respectiva área.

Os valores mínimos obtidos nos QD postural e linguagem (Tabela 2) tratam de um lactente nascido de parto normal, com idade gestacional de 36 semanas e peso ao nascer de $2.210 \mathrm{~g}$, que não necessitou de procedimento de urgência durante o parto, porém ao nascer apresentou diagnóstico de sífilis congênita. Em se tratando dos resultados da Escala Brunet-Lèzini, observou-se classificação inferior para a área da linguagem constatando-se atraso no desenvolvimento e alerta para atraso na área postural e de sociabilidade. A sífilis congênita pode levar a abortamento espontâneo, morte fetal e neonatal, prematuridade e danos à saúde do recém nascido com repercussōes psicológicas, motoras e sociais. Além disso, o lactente pode apresentar atraso no desenvolvimento da linguagem e da cognição ${ }^{26}$.
Os valores mínimos obtidos na área de desenvolvimento da coordenação óculo-motriz e global (Tabela 2) referem-se a um lactente nascido de parto normal, com idade gestacional de 35 semanas e peso ao nascer de $1.740 \mathrm{~g}$, que não necessitou de procedimento de urgência durante o parto e nem apresentou complicaçóes logo após o nascimento, porém apresentou classificação inferior na área de desenvolvimento da coordenação óculo-motriz e global caracterizando atraso, sendo as demais áreas classificadas em normal médio. Supóe-se que fatores ambientais possam estar interferindo no desenvolvimento deste lactente, já que não há relato de fatores biológicos cumulativos. Os fatores ambientais envolvem os cuidados precários à saúde, fatores socioeconômicos, ambiente familiar desfavorável, tabagismo, exposição a drogas, assistência pré-natal inadequada, acidentes e violência ${ }^{2}$.

Ressalta-se no estudo que, entre os lactentes que apresentaram classificação normal baixo, 3 eram gêmeos, sendo dois destes irmãos. Estes lactentes obtiveram alerta para atraso na área da coordenação óculo-motriz, sendo as demais áreas classificadas dentro da normalidade. Os gêmeos monozigóticos e dizigóticos podem ser expostos a fatores de risco para problemas pré e peri-natais, como compressão intra-uterina, traumas e prematuridade, os quais podem ocasionar lesão neurológica e atraso no desenvolvimento neuropsicomotor ${ }^{27}$.

Já em relação aos QD da Tabela 3, referente à faixa etária de 8 a 10 meses e 29 dias, observa-se também que todas as áreas psicomotoras encontram-se dentro da normalidade. O nível motor da área da coordenação óculo-motriz, social, linguagem e global foi classificado como normal médio. Já a área postural apresentou classificação normal alto. Entretanto, os valores mínimos dos QD revelam classificação inferior em todas as áreas de desenvolvimento.

Os valores mínimos obtidos nas áreas de desenvolvimento postural, linguagem e sociabilidade (Tabela 3) referem-se ao lactente que apresentou peso de $1.790 \mathrm{~g}$ e idade gestacional de 35 semanas, Apgar igual a 7 no primeiro minuto de vida e igual a 9 no quinto minuto. A mãe relatou uma ocorrência gestacional (ameaça de aborto), necessidade de urgência para respirar logo após o nascimento, complicações respiratórias e convulsóes neonatais, necessitando permanecer internado na Unidade de Terapia Intensiva durante 20 dias logo após o 
nascimento. Em se tratando dos resultados da Escala Brunet-Lèzini, observou-se classificação inferior para todas as áreas de desenvolvimento, constatando-se atraso no desenvolvimento neuropsicomotor global. $\mathrm{Na}$ avaliação deste lactente identificou-se aumento de tônus muscular em membros superiores e inferiores, sendo estes mais afetados que os superiores, sugerindo um quadro de diparesia espástica. Bebês prematuros são particularmente mais vulneráveis a processos isquêmicos e hemorrágicos no período intraparto e neonatal. Nestes casos, a Paralisia Cerebral pode representar o ponto final de uma cadeia de efeitos adversos, ocorrendo em um período em que o cérebro está particularmente vulnerável a danos de origem vascular $^{28}$.

O valor mínimo obtido no QD na área postural (Tabela 3) trata-se de um lactente nascido de parto normal, gemelar, com idade gestacional de 35 semanas e peso ao nascer de $1.935 \mathrm{~g}$ que náo necessitou de procedimento de urgência durante o parto e não apresentou complicações logo após o nascimento. Porém, apresentou classificação inferior na área de desenvolvimento postural, caracterizando atraso, e classificação normal baixo nas áreas de desenvolvimento social e global, demonstrando alerta para atraso. A avaliação do irmão gêmeo deste lactente com peso ao nascer de $1.920 \mathrm{~g}$ evidenciou alerta para atraso na área da coordenação óculo-motriz. Corroborando com este achado, uma pesquisa realizada com lactentes prematuros, que teve por objetivo avaliar o risco neurológico para o desenvolvimento, constatou que a área da coordenação óculo-motriz apresentou os menores escores na classificação do desenvolvimento, seguida pelas demais áreas ${ }^{18}$.

O valor mínimo do QD global (Tabela 3) refere-se a um lactente nascido de parto cesáreo, gemelar, com idade gestacional de 32 semanas e peso ao nascer de $1.730 \mathrm{~g}$ que necessitou de medidas de urgência para respirar e apresentou complicaçóes respiratórias logo após o nascimento. Neste caso detectou-se atraso no desenvolvimento nas áreas postural, da coordenação óculo-motriz, da sociabilidade e global. $\mathrm{Na}$ área da linguagem observou-se alerta de atraso.

Outro caso que chama atenção no estudo é o de um lactente nascido de parto cesáreo com idade gestacional de 36 semanas e peso ao nascer de $2.250 \mathrm{~g}$ que apresentou ocorrência gestacional do tipo pressão alta, necessitou de medidas de urgência para respirar e apresentou complicaçóes respiratórias e cardíacas logo após o nascimento. $\mathrm{Na}$ avaliação deste lactente constatou-se atraso nas áreas de desenvolvimento postural e social e alerta para atraso nas áreas de linguagem e no desenvolvimento global. A hipertensão gestacional é considerada indicador de risco para o desenvolvimento neuropsicomotor de lactentes. Lactentes provenientes de partos que tiveram ocorrência gestacional do tipo pressão alta podem nascer prematuros, com baixo peso, apresentar distúrbios e assimetria da motricidade voluntária e hipotonia ${ }^{20}$. A prematuridade associada com problemas respiratórios no período perinatal decorrente de complicaçóes no parto pode comprometer o DNPM, aumentando o risco para atraso no desenvolvimento dos lactentes ${ }^{5}$.

Uma das principais finalidades do seguimento dos lactentes prematuros é a identificação precoce de sinais de anormalidades do desenvolvimento, desta forma a intervenção em tempo oportuno se faz necessária ${ }^{2}$. Neste sentido, avaliar o DNPM ainda no primeiro ano de vida, principalmente nas situações de risco biológico e/ou ambiental, é um aspecto determinante no acompanhamento de lactentes que se encontram em situação de vulnerabilidade ${ }^{29}$.

\section{CONCLUSÃO}

Em ambas as faixas etárias avaliadas, a área de desenvolvimento que mais se destacou, em termos de alerta para atraso ou atraso já instalado, foi a coordenação óculo-motriz, já que 5 lactentes da faixa etária de 6 a 7 meses e 29 dias e 5 na faixa etária de 8 a 10 meses e 29 dias apresentaram perfis com classificação normal baixo ou inferior. Este achado indica a necessidade de açóes preventivas voltadas para a estimulação do neurodesenvolvimento, com especial atenção para a coordenação óculo-motriz. Orientações para as mães quanto à importância do manuseio e exploração de brinquedos e objetos adequados à idade corrigida do pré-termo são ações em saúde que podem contribuir para o potencial de desenvolvimento destes lactentes.

Observou-se em nosso estudo que a maioria dos lactentes que apresentaram sinais de alerta ou atraso já instalado no DNPM necessitaram de medidas de urgência para respirar e desenvolveram, com mais frequência, 
complicações respiratórias logo após o parto, sendo estas condiçóes biológicas de risco que foram somadas à prematuridade e ao baixo peso.

Os resultados encontrados reforçam a necessidade de identificar precocemente os sinais de atrasos no desenvolvimento de lactentes vulneráveis, possibilitando o encaminhamento e a intervenção precoce. Um programa de seguimento pós-alta, estruturado por uma equipe multiprofissional que conheça os riscos a que as crianças foram expostas, oferece condiçôes de acompanhamento adequado, já que a avaliação sistemática do desenvolvimento possibilita detectar precocemente as alteraçóes e intervir em tempo oportuno.

\section{REFERÊNCIAS}

1.Oliveira CEN, Salina ME, Annunciato NF. Fatores ambientais que influenciam a plasticidade do sistema nervoso central. Acta Fisiatr 2001;8:6-13. 2.Miranda LP, Resegue R, Figueiras ACM. A criança e o adolescente com problemas do desenvolvimento no ambulatório de pediatria. J Pediatr 2003;79:33-42. http://dx.doi.org/10.1590/S0021-75572003000700005

3.Eickmann SH, Lira PIC, Lima MC. Desenvolvimento mental e motor aos 24 meses de crianças nascidas a termo com baixo peso. Arq Neuropsiquiatr 2002;60:748-54.

http://dx.doi.org/10.1590/S0004-282X2002000500013

4.Halpern R, Giugliani ERJ, Victora CG, Barros FC, Bernardo LH. Fatores de risco para suspeita de atraso no desenvolvimento neuropsicomotor aos 12 meses de vida. J Pediatr 2000;76:421-8.

5.Tecklin JS. Fisioterapia pediátrica. 3 ed. Porto Alegre: Artmed, 2002, 480p. 6.Ramos SRP, Gugisch RC, Fraiz FC. The influence of gestational age and birth weight of the newborn on tooth eruption. J Appl Oral Sci 2006;14:228-32. http://dx.doi.org/10.1590/S1678-77572006000400003

7.Meio MDBB, Lopes CS, Morsch DS. Fatores prognósticos para o desenvolvimento cognitivo de prematuros de muito baixo peso. Rev Saúde Pública 2003;37:311-8.

8.Rugolo LMSS. Crescimento e desenvolvimento a longo prazo do prematuro extremo. J Pediatr 2005;81:101-10.

http://dx.doi.org/10.2223/JPED.1309

9.Saccani R, Brizola E, Giordani AP, Bach S, Resende TL, Almeida CS. Avaliaçáo do desenvolvimento neuropsicomotor em crianças de um bairro da periferia de Porto Alegre. Sci Med 2007;17:130-7.

10.Souza JM. Avaliaçáo do desenvolvimento neuropsicomotor de crianças de 6 a 24 meses matriculadas em creches municipais de Florianópolis/SC [dissertação mestrado]. Florianópolis: Universidade do Estado de Santa Catarina, 2003, 138p.
11.Brunet O, Lézine I. Desenvolvimento Psicológico da Primeira Infância. Porto Alegre: Artes Médicas, 1981, 159p.

12.Macdonald MG, Seshia MMK, Mullett MD. Neonatologia: fisiopatologia e tratamento do recém-nascido. In: Thompson MW, Hunt CE. Controle da respiraçấo: desenvolvimento, apnéia da prematuridade, eventos aparentes ameaçadores à vida, síndrome de morte súbita do lactente. Rio de Janeiro: Guanabara Koogan, 2007, p.356-75.

13. Castro GGD. Caracterização das crianças prematuras nascidas na cidade de Patrocínio (MG), no ano de 2004: Uma contribuição da Fisioterapia para o Programa de Saúde das crianças [dissertação mestrado]. Franca: Universidade de Franca; 2005, 65p.

14.Marcondes E. Pediatria básica. 9 ed. São Paulo: Sarvier, 2004, 843p.

15.Facchini FP, Mezzacapa MA, Rosa IRM, Filho FM, Netto AA, Marba STM. Acompanhamento da icterícia neonatal em recém-nascidos de termo e prematuros tardios. J Pediatr 2007;83:313-8.

http://dx.doi.org/10.1590/S0021-75572007000500005

16. Tronchin DMR, Tsunechiro MA. Prematuros de muito baixo peso: do nascimento ao primeiro ano de vida. Rev Gaúcha Enferm 2007;28:79-88.

17.Carbonero C, Alonso P. Seguimiento del prematuro/gran prematuro en atención primaria. Rev Pediatr Aten Primaria 2009;6:443-50.

18.Goyen TA, Lui K. Longitudinal motor development of "apparently normal" high-risk infants at 18 months, 3 and 5 years. Early Hum Dev 2002;70:03-115. 19.Linhares MBM, Carvalho AEV, Bordin MBM, Chimello JT, Martinez FE, Salim M. Prematuridade e muito baixo peso como fator de risco ao desenvolvimento da criança. Paidéia 2000;10:60-9.

20.Dias BR, Piovesana AMSG, Montenegro MA, Guerreiro MM. Desenvolvimento Neuropsicomotor de lactentes filhos de mães que apresentaram hipertensão arterial na gestação. Arq Neuropsiquiatr 2005;63:632-6.

21.Bittar RE, Zugaib M. Indicadores de risco para o parto prematuro. Rev Bras Ginecol Obstet 2009;31:203-9.

22.Rades E, Bittar RE, Zugaib M. Determinantes diretos do parto prematuro eletivo e os resultados neonatais. Rev Bras Ginecol Obstet 2004;26:655-62. http://dx.doi.org/10.1590/S0100-72032004000800010

23.Neto OLM, Barros MBA. Fatores de risco para mortalidade neonatal e pósneonatal na Regiáo Centro-Oeste do Brasil: linkage entre bancos de dados de nascidos vivos e óbitos infantis. Cad Saúde Pública 2000;16:477-85.

24.Ramos JG, Costa SM, Filho JSC, Souza C, Castilhos MF. Fabíola. Complicaçōes da gemelaridade em um hospital universitário. Rev HCPA 2000;20:114-8. 25.Mello RR, Dutra MVP, Lopes JMA. Morbidade respiratória no primeiro ano de vida de prematuros egressos de uma unidade pública de tratamento intensivo neonatal. J Pediatr 2004;80:503-10.

http://dx.doi.org/10.2223/1265

26.Rodrigues CS, Guimarães MDC. Grupo Nacional de estudo sobre Sífilis Congênita. Positividade para sífilis em puérperas: ainda um desafio para o Brasil. Rev Panam Salud Publica 2004;16:168-75.

http://dx.doi.org/10.1590/S1020-49892004000900003

27.Cintas HL. Crescimento e Desenvolvimento. In: Long TM, Cintas HL. Manual de fisioterapia pediátrica. Rio de Janeiro: Revinter; 2001, p.1-56.

28.Airoldi MJ, Silva SBDC, Souza RCT. Avaliação de recém-nascidos prétermo com hemorragia peri-ventricular e/ou leucomalácia periventricular. Rev Neurocienc 2009;17:24-9.

29.Nascimento R, Piassão C. Avaliação e estimulação do desenvolvimento neuropsicomotor em lactentes institucionalizados. Rev Neurocienc 2010;18:469-78. 